\title{
The overlearning reversal effect: A function of the non-monotonicity of the negativity of S- during discriminative training'
}

GERALD B. BIEDERMAM

UNIVERSITY OF TORONTO

In an automatic visual discrimination setting, rats learned two discriminations at once, one of which received four times the training of the other. After training, $S$ could be given his choice between the more-trained (MT) and less-trained (LT) negative stimuli (S-). S's choice of the MT S-indicates that its negativity was lower than that of the LT S-. In control groups. MTS + was consistently chosen over LTS + An explanation of these findings suggests that $S$-provides the initial cue function in discrimination. Later in training, $S_{+}$ presumably assumes the cue function.

The overlearning reversal effect (ORE) has enjoyed much recent experimental and theoretical attention. The product of this labor is an array of contradictory data and an attendant confusion in theory. It will be the purpose of this paper to show that confusion has derived from the inappropriateness of at least one basic assumption and the lack of an experimental design to detect this shortcoming.

The ORE is itself easily described; rats receiving considerable overtraining reverse faster than Ss reversed after just reaching a learning criterion. Lovejoy (1966) has outlined the three assumptions of reinforcement theorists that cause the ORE to appear paradoxical:

"(a) Habit strength increases monotonically with the number of reinforcements; (b) the greater the habit strength, the greater the resistance to extinction; (c) reversal learning is simply a matter of extinguishing an old habit and then learning the opposite one. From these three assumptions, theorists have properly deduced that the ORE should not occur"' (Lovejoy, 1966, p. 88).

D'Amato \& Jagoda (1962) have in fact proposed that the assumption of the monotonicity of habit strength with respect to number of reinforcements is unsatisfactory. Specifically, D'Amato and Jagoda offer the possibility that the aversiveness of the negative stimulus (S-) does not increase monotonically as learning proceeds. They suppose that this decrement in negativity of Sis produced by the decreasing experience $S$ has with $S-$ in overtraining.

It was the purpose of a recent study (Deutsch \& Biederman, 1965) to develop a method by which the negativity curve of the $S-$ could be evaluated without using forcing procedures. The data from this study support the hypothesis that the $\mathrm{S}-$ is not monotonic in its negativity.
The experiments described in the present paper were designed in order to verify and extend the data provided by Deutsch \& Biederman (1965) using a totally automated experimental setting. A second purpose of the present study was to evaluate the acquisition of the positivity of St.

Method

Sixty-three Long-Evans adult male rats served as Ss. A 23-hr. water deprivation schedule, food ad lib, was initiated for each $\mathrm{S}$ seven days before the beginning of training.

Training took place in a two-bar experimental chamber with a visual display in "+" shape over each bar. A water dipper was located between the levers. A reward light was placed between and above the two displays. Each visual display consisted of nine pilot lights, two in each arm of the " + " and one central light. The lights were so arranged that the horizontal and vertical arms were independently energized. The central pilot light in each display was L.dependently powered and could be lighted at either of two intensities. This arrangement provided $S$ with two simultaneous discriminations, horizontal vs. vertical lights, and bright vs. dim. The programming device is described in detail elsewhere (Deutsch \& Biederman, 1966). Reward or its absence, which depended on the accuracy of the response, and test trial were programmed automatically.

Ss were randomly assigned to one of the two training groups, receiving 64 training trials (Group 64) or 128 training trials (Group 128). In Group 64, 22 Ss were given a choice between the negative stimuli in the critical test following training while the remaining 17 were given a choice between the positive stimuli from the $M T$ and LT discriminations. In Group 128, 12 of the $42 \mathrm{Ss}$ used were presented with the positive stimuli on the test trial, and 12 were presented with the negative stimuli. MT and LT discriminations were in the ratio of $4: 1$.

On Day 1, each $S$ was shaped to press each lever to receive water. On Day 2, each $S$ received discriminative training. After the last trial the test pairing was presented. A 15-sec. intertrial interval occurred after each response. During this period, all displays were unlit and the manipulanda were deactivated.

Resulis

The test pairing of the MT and LT negative stimuli produced the following results: in the 64 trial group 18 of 22 Ss chose the MT S-. The one-tailed probability 


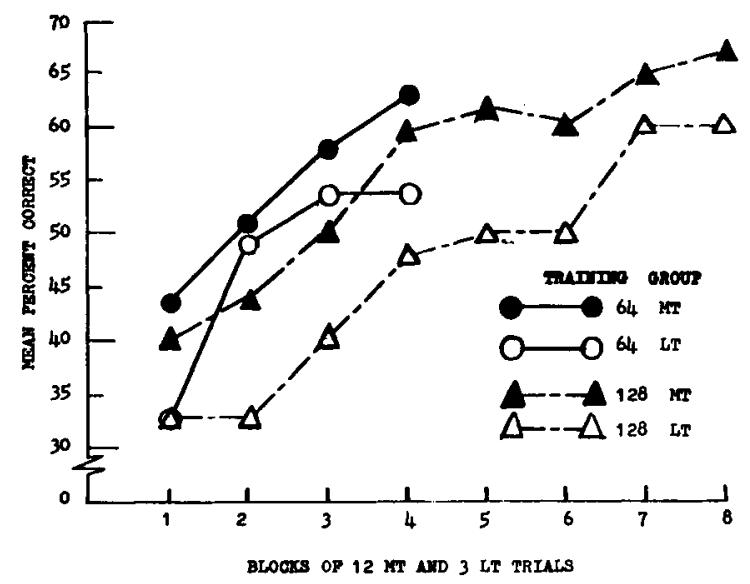

Fig. 1. The acquisition of MT and LT discriminations.

of this ratio occurring by chance is $\mathbf{. 0 0 2}$. In the 128 trial group, seven of $12 \mathrm{Ss}$ chose the MT S-. This ratio is the same as in Deutsch \& Blederman's (1965) group which received 132 training trials and occurs by chance $40 \%$ of the time (one-tailed probability). Fifteen of 17 Ss choosing between the MT S+ and the LT S+ in Group 64 chose the MT $S+(p=.001)$. In Group 128, 10 of $12 \mathrm{Ss}$ chose the MT $S+(p=.02)$. The acquisition functions for the MT and LT discriminations are presented in Fig. 1. The acquisition functions obtained are similar to those of typical group learning curves which may be described as negatively accelerated monotonic functions.

In Group 64, in the MT discrimination, the trials main effect was significant, $F=6.65, d f=3 / 105, p<.005$, as was the linear trend, $F=19.94, \mathrm{df}=1 / 105, \mathrm{p}<.05$. The LT discrimination for Group 64 produced a significant trials main effect, $F=4.80, d f=3 / 105, p<.005$. The linear trend was again highly significant, $F=10.64$, df $=1 / 105, p<.005$.

In Group 128 the same pattern emerged. In the MT discrimination the trials main effect was significant, $F=10.99$, df $=7 / 161, p<.005$, and the linear trend was also highly significant, $F=71.88, \mathrm{df}=1 / 161, p<.005$. The LT discrimination in Group 128 had a significant trials main effect, $F=5.77$, df $=7 / 161, p<.005$. Here the linear trend was again highly significant, $F=37.95, d f=1 / 161$, $p<.005$.

\section{Discussion}

The results of this study show that the assumption that the aversiveness of the negative stimulus in simultaneous discrimination is monotonic over trials, can no longer serve as a basls for an explanation of the ORE.
The negativity curve for S- in simultaneous discrimination may be viewed as a two-step process. Initially the negativity curve of S- is high, but as training proceeds this function changes so that $S-$ no longer serves as the effective cue in discrimination and therefore begins to lose its negativity. In Group 64, for example, 18 of 22 Ss selected the MT S-. Here, Ss had received about 51 MT and 13 LT discrimination trials. By the time 128 trials had elapsed for Group 128, with 102 MT and 26 LT trials, the choice approached chance levels. This random choice may occur because the LT discrimination now begins to join the MT discrimination in having $\mathrm{S}+$ as its primary cue.

The ORE is obtained (with some exceptions) in visual discrimination but not in position discrimination. In the latter, there are many unnecessary but correlated cues associated with S+ and S-. It is difficult in this instance to ascertain which cues subserve the discriminative performance. $\mathrm{S}$ may shift from trial to trial in attention to various cues in learning such a discrimination. The failure of the ORE to occur in position discrimination may be understood if the mechanism that switches $S$ from avoidance of $S$ - early in training to approach of $S+$ later in training is retarded by the multiplicity of cues as $\mathrm{S}+$ or $\mathrm{S}$ - in position discrimination. In D'Amato \& Schiff's (1965) experiments, where the ORE was not obtained, although the discrimination was visual, the use of automated $Y$ mazes that cause the goal and start positions to interchange after each trial might have been disruptive.

\section{References}

Biederman, G. B. The overlearning reversal effect: a function of the non-monotonicity of the negativity of $S$-during discriminative training. Ann Arbor, Michigan: University Microfilms, 1966.

D'Amato, M. R., \& Jagoda, $\mathbf{H}$. Overlearning and position reversal. J. exp. Psychol., 1962, 64, 117-122.

D'Amato, M. R., \& Schiff, D. Overlearning and brightness discrimination reversal. J. exp. Psychol., 1965, 69, 375-381.

Deutsch, J. A., \& Biederman, G. B. The monotonicity of the negative stimulus during learning. Psychon. Sci., 1965, 3, 391-392.

Deutsch, J. A., \& Biederman, G. B. A multiple discrimination computer. Med. Biol. Engng., 1967, in press.

Lovejoy, E. Analysis of the overlearning reversal effect. $P$ sychol. Rev., 1966, 73, 87-103.

\section{Note}

1. This paper is based on a dissertation submitted in partial fulfillment of the requirements for the Ph.D. degree at New York University, (Biederman, 1966). The author gratefully acknowledges the guidance of Professor J. Anthony Deutsch, Chairman of the Dissertation Committee. The research was carried out while the author was a Predoctoral Fellow of the National Institutes of Health. 